\title{
Integral geometry of pairs of hyperplanes or lines
}

\author{
Daniel Hug And Rolf Schneider
}

\begin{abstract}
Crofton's formula of integral geometry evaluates the total motion invariant measure of the set of $k$-dimensional planes having nonempty intersection with a given convex body. This note deals with motion invariant measures on sets of pairs of hyperplanes or lines meeting a convex body. Particularly simple results are obtained if, and only if, the given body is of constant width in the first case, and of constant brightness in the second case.
\end{abstract}

Mathematics Subject Classification. Primary 52A20, Secondary 53C65.

Keywords. Crofton formula, Invariant measure, Constant width, Constant brightness.

1. Introduction. More than 150 years ago, Crofton [1] proved that the total motion invariant measure of the set of lines meeting a given convex body $K$ in the Euclidean plane is equal to the boundary length $L(K)$ of $K$, multiplied by a factor that depends only on the normalization of the measure. Nowadays, a generalization of this result, known as 'Crofton's formula' in integral geometry, may be written as

$$
\int_{A(d, k)} V_{0}(K \cap E) \mu_{k}(\mathrm{~d} E)=c_{d k} V_{d-k}(K), \quad k=1, \ldots, d-1,
$$

for $K \in \mathcal{K}^{d}$, the set of convex bodies (nonempty compact convex sets) in Euclidean space $\mathbb{R}^{d}$. Here $A(d, k)$ is the space of $k$-dimensional affine subspaces of $\mathbb{R}^{d}$ with its usual topology, $\mu_{k}$ is its motion invariant measure with a suitable normalization, and the constant $c_{d k}$ depends on this normalization. We refer to $[8,(4.59)]$ or $[9$, Thm. 5.1.1] for more details and a more general formula. The functionals $V_{0}, \ldots, V_{d-1}$ are the intrinsic volumes, which can be defined by the Steiner formula 


$$
V_{d}\left(K+\lambda B^{d}\right)=\sum_{k=0}^{d} \lambda^{d-k} \kappa_{d-k} V_{k}(K), \quad \lambda \geq 0
$$

where $V_{d}$ denotes the volume, $B^{d}$ is the unit ball of $\mathbb{R}^{d}$, and $\kappa_{d}=V_{d}\left(B^{d}\right)$ (see, e.g., [8, Section 4.1]). In particular, $V_{0}(K)=1$ for every convex body $K$, and if one extends this by defining $V_{0}(\emptyset)=0$, then $V_{0}=\chi$ is the Euler characteristic on $\mathcal{K}^{d} \cup\{\emptyset\}$. Further, $c_{d} V_{1}=W$ with $c_{d}=2 \kappa_{d-1} /\left(d \kappa_{d}\right)$ is the mean width, and $2 V_{d-1}=S$ is the surface area. With the usual normalization, we can write two special cases of the Crofton formula as

$$
\int_{A(d, d-1)} \chi(H \cap K) \mu_{d-1}(\mathrm{~d} H)=W(K)
$$

and

$$
\int_{A(d, 1)} \chi(G \cap K) \mu_{1}(\mathrm{~d} G)=2 c_{d} S(K) .
$$

In the plane, both formulas (1) and (2) yield the same, namely Crofton's original formula.

Recently, Cufí, Gallego, and Reventós [2] have computed certain motion invariant measures of pairs of lines meeting a planar convex body. They consider measures on pairs of lines in the plane which with respect to the product $\mu_{1} \otimes \mu_{1}$ of the invariant line measure $\mu_{1}$ have a density that depends only on the angle between the lines. More precisely, let $f: \mathbb{R} \rightarrow \mathbb{R}$ be a function which is even, $\pi$-periodic, and integrable over $[0, \pi]$. For a line $G$ in the plane, let $\varphi(G)$ be the angle that it makes with a fixed direction. Then the article [2] treats (with different notation) the integral

$$
I(K, f):=\int_{A(2,1)} \int_{A(2,1)} \chi\left(G_{1} \cap K\right) \chi\left(G_{2} \cap K\right) f\left(\varphi\left(G_{1}\right)-\varphi\left(G_{2}\right)\right) \mu_{1}\left(\mathrm{~d} G_{1}\right) \mu_{1}\left(\mathrm{~d} G_{2}\right) .
$$

The authors express this integral in terms of the Fourier coefficients of $f$ and of the support function of $K$. While aiming at various consequences, they note that for a body $K$ of constant width, one has the simple formula

$$
I(K, f)=\lambda[f] L(K)^{2},
$$

where the constant $\lambda[f]$ depends only on $f$, and hence is given by $\lambda[f]=$ $I\left(B^{2}, f\right) /\left(4 \pi^{2}\right)$. We recall that the width of a convex body $K \in \mathcal{K}^{d}$ at a unit vector $u$ is the distance of the two supporting hyperplanes of $K$ orthogonal to $u$.

In the following, we extend the preceding observations to higher dimensions, in two different ways, considering either hyperplanes or lines. We prove also a converse to the higher-dimensional version of (3). A main goal is to assume only invariance properties of the underlying measures, and not a specific analytic representation involving a density with respect to $\mu_{k} \otimes \mu_{k}$. To make this precise, we recall that $A(d, k)$ is the space of $k$-dimensional planes in $\mathbb{R}^{d}$, with its usual topology, and by $G(d, k)$ we denote the Grassmannian of $k$-dimensional linear subspaces of $\mathbb{R}^{d}$ (for $k \in\{1, \ldots, d-1\}$ ). For a topological space $X$, we denote by 
$\mathcal{B}(X)$ the $\sigma$-algebra of Borel subsets of $X$. Measures in the following, without further specification, are Borel measures. Let $\mu$ be a measure on $A(d, k)^{2}$. The measure $\mu$ is called separately translation invariant if for any $B \in \mathcal{B}\left(A(d, k)^{2}\right)$ and $x_{1}, x_{2} \in \mathbb{R}^{d}$, the relation $\mu\left(\left\{\left(L_{1}+x_{1}, L_{2}+x_{2}\right):\left(L_{1}, L_{2}\right) \in B\right\}\right)=\mu(B)$ is satisfied. We denote by $\mathrm{G}(d)$ the group $\mathrm{SO}(d)$ if $d$ is even and the group $\mathrm{O}(d)$ if $d$ is odd. The measure $\mu$ is called jointly $\mathrm{G}(d)$-invariant if for any $B \in \mathcal{B}\left(A(d, k)^{2}\right)$ and any $\vartheta \in \mathrm{G}(d)$, we have $\mu(\vartheta B)=\mu(B)$, where $\vartheta B:=$ $\left\{\left(\vartheta L_{1}, \vartheta L_{2}\right):\left(L_{1}, L_{2}\right) \in B\right\}$. A similar definition is used for measures on $G(d, k)^{2}$ or on $\left(\mathbb{S}^{d-1}\right)^{2}$, where $\mathbb{S}^{d-1}$ is the unit sphere. By $\mathcal{M}_{k}$ we denote the set of locally finite Borel measures on $A(d, k)^{2}$ which are separately translation invariant, jointly $\mathrm{G}(d)$-invariant, and symmetric, that is, invariant under the mapping $\left(L_{1}, L_{2}\right) \mapsto\left(L_{2}, L_{1}\right)$.

More generally, we can consider two convex bodies $K_{1}, K_{2} \in \mathcal{K}^{d}$. Let $\Theta$ be a locally finite measure on the space $A(d, d-1)^{2}$ of pairs of hyperplanes. Then we define

$$
I\left(K_{1}, K_{2}, \Theta\right):=\Theta\left(\left\{\left(H_{1}, H_{2}\right) \in A(d, d-1)^{2}: H_{i} \cap K_{i} \neq \emptyset(i=1,2)\right\}\right) .
$$

Similarly, with a locally finite measure $\Theta$ on the space $A(d, 1)^{2}$ of pairs of lines, we define

$$
J\left(K_{1}, K_{2}, \Theta\right):=\Theta\left(\left\{\left(G_{1}, G_{2}\right) \in A(d, 1)^{2}: G_{i} \cap K_{i} \neq \emptyset(i=1,2)\right\}\right) .
$$

We write $I(K, \Theta):=I(K, K, \Theta)$ and $J(K, \Theta):=J(K, K, \Theta)$.

If $\Theta \in \mathcal{M}_{i}$ for $i=d-1$, respectively $i=1$, general expressions for the quantities $I\left(K_{1}, K_{2}, \Theta\right), J\left(K_{1}, K_{2}, \Theta\right)$ will be given in Theorem 6 . This theorem requires some preparations, therefore it will be formulated only in Section 4. Already here we can state the following.

Theorem 1. Let $K_{1}, K_{2} \in \mathcal{K}^{d}$, and let $\Theta$ be a locally finite measure on $A(d, d-$ $1)^{2}$ which is separately translation invariant. If $K_{1}, K_{2}$ are bodies of constant width, then

$$
I\left(K_{1}, K_{2}, \Theta\right)=\lambda[\Theta] W\left(K_{1}\right) W\left(K_{2}\right) \quad \text { with } \lambda[\Theta]:=I\left(B^{d}, \Theta\right) / 4 .
$$

The following theorem shows that convex bodies of constant width necessarily enter the scene in this situation.

Theorem 2. If a convex body $K \in \mathcal{K}^{d}$ satisfies

$$
I(K, \Theta)=\lambda[\Theta] W(K)^{2} \quad \text { with } \lambda[\Theta]:=I\left(B^{d}, \Theta\right) / 4
$$

for each measure $\Theta \in \mathcal{M}_{d-1}$, then $K$ has constant width.

As the proof will show, relation (5) is only required for a subclass of the set of measures in $\mathcal{M}_{d-1}$. A similar remark applies to Theorem 4 .

Instead of affine subspaces of codimension one, we can also consider affine subspaces of dimension one. In that case, the role of the width is taken over by the brightness. The brightness of a convex body $K \in \mathcal{K}^{d}$ at a unit vector $u$ is the $(d-1)$-dimensional volume of the orthogonal projection of $K$ to a hyperplane orthogonal to $u$. 
Theorem 3. Let $K \in \mathcal{K}^{d}$, and let $\Theta$ be a locally finite measure on $A(d, 1)^{2}$ which is separately translation invariant. If $K_{1}, K_{2}$ are bodies of constant brightness, then

$$
J\left(K_{1}, K_{2}, \Theta\right)=\kappa[\Theta] S\left(K_{1}\right) S\left(K_{2}\right) \quad \text { with } \kappa[\Theta]:=J\left(B^{d}, \Theta\right) / S\left(B^{d}\right)^{2} .
$$

Theorem 4. If a convex body $K \in \mathcal{K}^{d}$ satisfies

$$
J(K, \Theta)=\kappa[\Theta] S(K)^{2} \quad \text { with } \kappa[\Theta]:=J\left(B^{d}, \Theta\right) / S\left(B^{d}\right)^{2}
$$

for each measure $\Theta \in \mathcal{M}_{1}$, then $K$ has constant brightness.

If $\Theta \in \mathcal{M}_{d-1}$, then (4) holds already if only one of the two convex bodies is of constant width.

Theorem 5. Let $K_{1}, K_{2} \in \mathcal{K}^{d}$, and let $\Theta \in \mathcal{M}_{d-1}$. If $K_{1}$ is a body of constant width, then

$$
I\left(K_{1}, K_{2}, \Theta\right)=\lambda[\Theta] W\left(K_{1}\right) W\left(K_{2}\right) \quad \text { with } \lambda[\Theta]:=I\left(B^{d}, \Theta\right) / 4 .
$$

Again, there is also an analogous counterpart to equation (6), which we do not formulate.

Concerning bodies of constant width in general, we refer to the recent comprehensive monograph [5] by Martini, Montejano, and Oliveros. Information on bodies of constant brightness can be found in Gardner's book [3], in particular Section 3.2 and its notes.

That equation (4) holds for bodies of constant width and (6) holds for bodies of constant brightness, follows easily in the next section, once the separately translation invariant measures on $A(d, k)^{2}$ have been found to have a special form. Theorems 2, 4, and 5 will be proved in Section 4, after Theorem 6 has been treated. Before that, we need to find analytic representations for the measures under consideration; these will be established in the next two sections.

2. Separately translation invariant measures. The following lemma, which is formulated for general $k$, allows us to deal easily with translations. Here we denote by $\lambda_{L}$ the $j$-dimensional Lebesgue measure in a subspace $L \in G(d, j)$.

Lemma 1. Let $k \in\{1, \ldots, d-1\}$. Let $\Theta$ be a locally finite, separately translation invariant measure on $A(d, k)^{2}$. Then there exists a uniquely determined finite measure $\Theta_{0}$ on $G(d, k)^{2}$ such that

$$
\Theta(A)=\int_{G(d, k)^{2}} \int_{L_{1}^{\perp}} \int_{L_{2}^{\perp}} \mathbb{1}_{A}\left(L_{1}+x_{1}, L_{2}+x_{2}\right) \lambda_{L_{2}^{\perp}}\left(\mathrm{d} x_{2}\right) \lambda_{L_{1}^{\perp}}\left(\mathrm{d} x_{1}\right) \Theta_{0}\left(\mathrm{~d}\left(L_{1}, L_{2}\right)\right)
$$

for every Borel set $A \subset A(d, k)^{2}$. If $\Theta$ is jointly $\mathrm{G}(d)$-invariant and symmetric, then $\Theta_{0}$ is jointly $\mathrm{G}(d)$-invariant and symmetric.

Proof. This can be shown in an elementary way by modifying the proof of [9, Thm. 4.4.1]. We reproduce part of the proof, to indicate the necessary modifications. 
We choose a $(d-k)$-dimensional subspace $U \in G(d, d-k)$, and define $G_{U}:=\{L \in G(d, k): \operatorname{dim}(L \cap U)=0\}, \quad A_{U}:=\left\{L+x: L \in G_{U}, x \in U\right\}$. The mapping $\varphi: G_{U}^{2} \times U^{2} \rightarrow A_{U}^{2},\left(L_{1}, L_{2}, x_{1}, x_{2}\right) \mapsto\left(L_{1}+x_{1}, L_{2}+x_{2}\right)$, is a homeomorphism. We fix $A \in \mathcal{B}\left(G_{U}^{2}\right)$, and for $B \in \mathcal{B}\left(U^{2}\right)$, we define $\eta(B):=\Theta(\varphi(A \times B))$. Then $\eta$ is a locally finite and translation invariant measure on $U^{2}$, hence it is a constant multiple of the product measure $\lambda_{U} \otimes \lambda_{U}$. Denoting the factor by $\rho(A)$, we thus have

$$
\Theta(\varphi(A \times B))=\rho(A)\left(\lambda_{U} \otimes \lambda_{U}\right)(B) .
$$

Evidently, $\rho$ is a finite measure on $G_{U}^{2}$. Thus, $\varphi^{-1}(\Theta)(A \times B)=\left(\rho \otimes \lambda_{U} \otimes\right.$ $\left.\lambda_{U}\right)(A \times B)$, where $\varphi^{-1}(\Theta)$ denotes the image measure of $\Theta\left\llcorner A_{U}^{2}\right.$ under the mapping $\varphi^{-1}$. This gives $\varphi^{-1}(\Theta)=\rho \otimes \lambda_{U} \otimes \lambda_{U}$ and, therefore, $\Theta\left\llcorner A_{U}^{2}=\right.$ $\varphi\left(\rho \otimes \lambda_{U} \otimes \lambda_{U}\right)$. Hence, for every nonnegative measurable function $f$ on $A(d, k)^{2}$, we have

$$
\begin{aligned}
\int_{A_{U}^{2}} f \mathrm{~d} \Theta & =\int_{G_{U}^{2} \times U^{2}}(f \circ \varphi) \mathrm{d}\left(\rho \otimes \lambda_{U} \otimes \lambda_{U}\right) \\
& =\int_{G_{U}^{2}} \int_{U^{2}} f\left(L_{1}+x_{1}, L_{2}+x_{2}\right) \lambda_{U}^{2}\left(\mathrm{~d}\left(x_{1}, x_{2}\right)\right) \rho\left(\mathrm{d}\left(L_{1}, L_{2}\right)\right) .
\end{aligned}
$$

For given $L \in G_{U}$, let $\Pi_{L}: U \rightarrow L^{\perp}$ denote the orthogonal projection to the orthogonal complement of $L$. It is bijective since $L \in G_{U}$. Therefore, $\Pi_{L}\left(\lambda_{U}\right)=a(L) \lambda_{L^{\perp}}$, with a factor $a(L)>0$ that depends only on $L$. Further, $L+x=L+\Pi_{L}(x)$. This yields

$$
\begin{aligned}
& \int_{U^{2}} f\left(L_{1}+x_{1}, L_{2}+x_{2}\right) \lambda_{U}^{2}\left(\mathrm{~d}\left(x_{1}, x_{2}\right)\right) \\
& \quad=a\left(L_{1}\right) a\left(L_{2}\right) \iint_{L_{1}^{\perp}} f\left(L_{1}+x_{1}, L_{2}+x_{2}\right) \lambda_{L_{2}^{\perp}}\left(\mathrm{d} x_{2}\right) \lambda_{L_{1}^{\perp}}\left(\mathrm{d} x_{1}\right) .
\end{aligned}
$$

Defining a measure $\Theta_{U}$ on $G_{U}^{2}$ by $a\left(L_{1}\right) a\left(L_{2}\right) \rho\left(\mathrm{d}\left(L_{1}, L_{2}\right)\right)=: \Theta_{U}\left(\mathrm{~d}\left(L_{1}, L_{2}\right)\right)$, we have

$$
\int_{A_{U}^{2}} f \mathrm{~d} \Theta=\iint_{G_{U}^{2}} \int_{L_{1}^{\perp}} \int_{L_{2}^{\perp}} f\left(L_{1}+x_{1}, L_{2}+x_{2}\right) \lambda_{L_{2}^{\perp}}\left(\mathrm{d} x_{2}\right) \lambda_{L_{1}^{\perp}}\left(\mathrm{d} x_{1}\right) \Theta_{U}\left(\mathrm{~d}\left(L_{1}, L_{2}\right)\right) .
$$

It is now clear from the rest of the proof of [9, Thm. 4.4.1] how one has to proceed to obtain the measure $\Theta_{0}$ satisfying (9).

From (9), we obtain, for $A \in \mathcal{B}\left(G(d, k)^{2}\right)$,

$$
\Theta_{0}(A)=\frac{1}{\kappa_{d-k}^{2}} \Theta\left(\left\{\left(L_{1}+x_{1}, L_{2}+x_{2}\right):\left(L_{1}, L_{2}\right) \in A, x_{i} \in B^{d}(i=1,2)\right\}\right) \text {. }
$$

From this equation, it is obvious that $\Theta_{0}$ is finite and uniquely determined. We also see that $\Theta_{0}$ is jointly $\mathrm{G}(d)$-invariant and symmetric if this holds for $\Theta$. 
For $K \in \mathcal{K}^{d}$ and $u \in \mathbb{S}^{d-1}$, let $w_{K}(u)$ be the width of $K$ at $u$. Moreover, for $H \in G(d, d-1)$, we denote by $u(H)$ one of the two unit normal vectors of $H$. If $K_{i} \in \mathcal{K}^{d}$ for $i=1,2$ and $\Theta$ satisfies the assumptions of Lemma 1, then this lemma yields

$$
\begin{aligned}
& I\left(K_{1}, K_{2}, \Theta\right)= \int_{G(d, d-1)^{2}} \int_{H_{1}^{\perp}} \int_{H_{2}^{\perp}} \mathbb{1}\left\{\left(H_{1}+x_{1}\right) \cap K_{1} \neq \emptyset\right\} \mathbb{1}\left\{\left(H_{2}+x_{2}\right) \cap K_{2} \neq \emptyset\right\} \\
& \lambda_{H_{2}^{\perp}}\left(\mathrm{d} x_{2}\right) \lambda_{H_{1}^{\perp}}\left(\mathrm{d} x_{1}\right) \Theta_{0}\left(\mathrm{~d}\left(H_{1}, H_{2}\right)\right) \\
&= \int_{G(d, d-1)^{2}} w_{K_{1}}\left(u\left(H_{1}\right)\right) w_{K_{2}}\left(u\left(H_{2}\right)\right) \Theta_{0}\left(\mathrm{~d}\left(H_{1}, H_{2}\right)\right)
\end{aligned}
$$

If now $K_{i}$ is of constant width $w_{K_{i}}=W\left(K_{i}\right)$ for $i=1,2$, then this gives

$$
I\left(K_{1}, K_{2}, \Theta\right)=W\left(K_{1}\right) W\left(K_{2}\right) \int_{G(d, d-1)^{2}} \Theta_{0}\left(\mathrm{~d}\left(H_{1}, H_{2}\right)\right),
$$

which is (4).

Relation (6) is obtained similarly, replacing the width function by the brightness function and noting that the surface area of a convex body is, up to a dimension-dependent factor, the mean value of its brightness function.

3. The measures in $\mathcal{M}_{\boldsymbol{d - 1}}$ or $\mathcal{M}_{\mathbf{1}}$. Our next aim is to obtain an analytic representation for jointly $\mathrm{G}(d)$-invariant measures on pairs of points on the unit sphere $\mathbb{S}^{d-1}$. By $\sigma$ we denote the spherical Lebesgue measure on $\mathbb{S}^{d-1}$. For $u \in \mathbb{S}^{d-1}$ and $t \in[-1,1]$, let $S_{u, t}:=\left\{x \in \mathbb{S}^{d-1}:\langle u, x\rangle=t\right\}$. For $t \in(-1,1)$, we denote by $\sigma_{u, t}$ the normalized spherical Lebesgue measure on the $(d-2)$ sphere $S_{u, t}$. For $t \in\{-1,1\}$, the measure $\sigma_{u, t}$ is the Dirac measure at $-u$, respectively $u$. The measures $\sigma_{u, t}$ are considered as measures on $\mathbb{S}^{d-1}$.

Lemma 2. Let $M$ be a finite, jointly $\mathrm{G}(d)$-invariant measure on $\left(\mathbb{S}^{d-1}\right)^{2}$. Then there is a unique finite, even measure $\psi$ on $[-1,1]$ such that

$$
\int_{\left(\mathbb{S}^{d-1}\right)^{2}} f \mathrm{~d} M=\int_{\mathbb{S}^{d-1}} \int_{[-1,1]} \int_{S_{u, t}} f(u, v) \sigma_{u, t}(\mathrm{~d} v) \psi(\mathrm{d} t) \sigma(\mathrm{d} u)
$$

for every nonnegative, measurable function $f$ on $\left(\mathbb{S}^{d-1}\right)^{2}$.

Proof. We use a result of Kallenberg on the existence of invariant disintegrations. It follows from [4, Thm. 3.5] (with $S=T=\mathbb{S}^{d-1}$ and $\nu:=M\left(\cdot \times \mathbb{S}^{d-1}\right)$ ) that $M=\nu \otimes \mu$ (which is explained in (13)), where $\mu$ is a $\mathrm{G}(d)$-invariant finite kernel from $\mathbb{S}^{d-1}$ to $\mathbb{S}^{d-1}$. We note that from $M=\nu \otimes \mu$ it follows that $\nu=\int \mathbb{1}\{s \in \cdot\} \mu(s, T) \nu(\mathrm{d} s)$, which implies that $\mu(s, T)=1$ for $\nu$-almost all $s \in S$. (A similar observation will be used below. In the present case, we may remark that, since $\mathrm{G}(d)$ acts transitively on $S$ and $\vartheta(T)=T$ for each $\vartheta \in \mathrm{G}(d)$, we even have $\mu(s, T)=1$ for all $s \in S$.) Since $\nu$ is a finite, rotation invariant Borel measure on $\mathbb{S}^{d-1}$, it is a constant multiple of the spherical Lebesgue mea- 
sure $\sigma$. Assuming that $M \neq \equiv$, we can choose $\nu=\sigma$, absorbing the constant into $\mu$. Then $M=\nu \otimes \mu$ means that

$$
\int_{\left(\mathbb{S}^{d-1}\right)^{2}} f \mathrm{~d} M=\int_{\mathbb{S}^{d-1}} \int_{\mathbb{S}^{d-1}} f(u, v) \mu(u, \mathrm{~d} v) \sigma(\mathrm{d} u)
$$

for every nonnegative, measurable function $f$ on $\left(\mathbb{S}^{d-1}\right)^{2}$. Here $\mu: \mathbb{S}^{d-1} \times$ $\mathcal{B}\left(\mathbb{S}^{d-1}\right) \rightarrow[0, \infty)$ is a kernel, that is, a mapping such that $\mu(u, \cdot)$ is a (finite) measure for each $u \in \mathbb{S}^{d-1}$ and $\mu(\cdot, A)$ is measurable for each $A \in \mathcal{B}\left(\mathbb{S}^{d-1}\right)$. The $\mathrm{G}(d)$-invariance of $\mu$ means that

$$
\mu(\vartheta u, \vartheta A)=\mu(u, A) \quad \text { for } u \in \mathbb{S}^{d-1}, A \in \mathcal{B}\left(\mathbb{S}^{d-1}\right), \vartheta \in \mathrm{G}(d) .
$$

We fix $u \in \mathbb{S}^{d-1}$ and define the map $p_{u}: \mathbb{S}^{d-1} \rightarrow[-1,1]$ by $p_{u}(v):=\langle u, v\rangle$. By $\psi_{u}=p_{u}(\mu(u, \cdot))$ we denote the image measure of $\mu(u, \cdot)$ under $p_{u}$.

First we show that $\psi_{u}$ is independent of $u$. Let $\vartheta \in \mathrm{G}(d)$. For $u, v \in \mathbb{S}^{d-1}$, we have $p_{\vartheta u}(v)=\langle\vartheta u, v\rangle=\left\langle u, \vartheta^{-1} v\right\rangle=p_{u}\left(\vartheta^{-1} v\right)$ and hence, for $A \in \mathcal{B}([-1,1])$,

$$
\begin{aligned}
x \in p_{\vartheta u}^{-1}(A) & \Leftrightarrow p_{\vartheta u}(x) \in A \Leftrightarrow p_{u}\left(\vartheta^{-1} x\right) \in A \Leftrightarrow \vartheta^{-1} x \in p_{u}^{-1}(A) \\
& \Leftrightarrow x \in \vartheta p_{u}^{-1}(A),
\end{aligned}
$$

thus $p_{\vartheta u}^{-1}(A)=\vartheta p_{u}^{-1}(A)$. This gives

$$
\psi_{\vartheta u}(A)=\mu\left(\vartheta u, p_{\vartheta u}^{-1}(A)\right)=\mu\left(\vartheta u, \vartheta p_{u}^{-1}(A)\right)=\mu\left(u, p_{u}^{-1}(A)\right)=\psi_{u}(A) .
$$

Therefore, we can from now on write $\psi_{u}=: \psi$.

By the independence just shown, and since the reflection in the origin is in $\mathrm{G}(d)$, we also have

$$
\begin{aligned}
\psi(-A) & =\psi_{-u}(-A)=\mu\left(-u, p_{-u}^{-1}(-A)\right)=\mu\left(-u,-p_{u}^{-1}(A)\right) \\
& =\mu\left(u, p_{u}^{-1}(A)\right)=\psi(A),
\end{aligned}
$$

thus the measure $\psi$ is even.

Now we further disintegrate the measure $\mu(u, \cdot)$. Let $q: \mathbb{S}^{d-1} \rightarrow \mathbb{S}^{d-1}$ denote the identity map. Then the image measure $M:=\left(p_{u} \times q\right)(\mu(u, \cdot))$ is a finite Borel measure on $[-1,1] \times \mathbb{S}^{d-1}$. We define the operations of the subgroup $\mathrm{G}_{u}(d):=\{\vartheta \in \mathrm{G}(d): \vartheta(u)=u\}$ of $\mathrm{G}(d)$ on $[-1,1]$ as the identity and on $\mathbb{S}^{d-1}$ in the usual way. Then it is easy to check that $\left(p_{u} \times q\right)(\mu(u, \cdot))$ is jointly invariant under $\mathrm{G}_{u}(d)$. Hence, by another application of Kallenberg's disintegration result (with $S=[-1,1], T=\mathbb{S}^{d-1}, \nu=M\left(\cdot \times \mathbb{S}^{d-1}\right)=\psi$ ), we obtain $\left(p_{u} \times q\right)(\mu(u, \cdot))=\psi \otimes \kappa_{u}$, where $\kappa_{u}:[-1,1] \rightarrow \mathbb{S}^{d-1}$ is a $\mathrm{G}_{u}(d)$-invariant kernel such that

$$
\int_{\mathbb{S}^{d-1}} h(\langle u, v\rangle, v) \mu(u, \mathrm{~d} v)=\int_{[-1,1] \mathbb{S}^{d-1}} \int_{1} h(t, v) \kappa_{u}(t, \mathrm{~d} v) \psi(\mathrm{d} t)
$$

for every nonnegative, measurable function $h$ on $[-1,1] \times \mathbb{S}^{d-1}$. As noted above, for $\psi$-almost all $t \in[-1,1]$, the measure $\kappa_{u}(t, \cdot)$ is a probability measure, and by (14), it is (for almost all $t$ ) concentrated on $S_{u, t}$ and invariant under $\mathrm{G}_{u}(d)$.

For $t \in(-1,1)$, this measure is supported by the sphere $S_{u, t}$, hence for $\psi$-almost all $t \in(-1,1)$, it is a constant multiple of the spherical Lebesgue measure on this sphere, thus $\kappa_{u}(t, \cdot)=c(u, t) \sigma_{u, t}$. Since $\kappa_{u}(t, \cdot)$ is a probability 
measure, $c(u, t)=c(t)$ is independent of $u$ for $\psi$-almost all $t \in(-1,1)$. With $c( \pm 1):=1$, the latter relation holds also for $t \in\{-1,1\}$. Since $\kappa_{u}\left(\cdot, \mathbb{S}^{d-1}\right)$ is measurable, this defines a measurable function $c(\psi$-almost everywhere on $[-1,1])$. In particular, for each nonnegative, measurable function $g$ on $\mathbb{S}^{d-1}$, we get

$$
\int_{\mathbb{S}^{d-1}} g \mathrm{~d} \mu(u, \cdot)=\int_{[-1,1]} \int_{S_{u, t}} g(y) \sigma_{u, t}(\mathrm{~d} y) c(t) \psi(\mathrm{d} t),
$$

and after redefining the measure $\psi$, we can write

$$
\int_{\mathbb{S}^{d-1}} g \mathrm{~d} \mu(u, \cdot)=\int_{[-1,1]} \int_{S_{u, t}} g(y) \sigma_{u, t}(\mathrm{~d} y) \psi(\mathrm{d} t) .
$$

Together with (13) (and $f(u, v)=g(v)$ ), this yields the assertion of the lemma.

The uniqueness of $\psi$ follows from (12) by choosing $f(u, v)=g(\langle u, v\rangle)$ with an arbitrary nonnegative, measurable function $g:[-1,1] \rightarrow[0, \infty)$. In fact, with this choice, we obtain

$$
\begin{aligned}
\int_{\left(\mathbb{S}^{d-1}\right)^{2}} g(\langle u, v\rangle) M(\mathrm{~d}(u, v)) & =\int_{\mathbb{S}^{d-1}} \int_{[-1,1]} \int_{S_{u, t}} g(\langle u, v\rangle) \sigma_{u, t}(\mathrm{~d} v) \psi(\mathrm{d} t) \sigma(\mathrm{d} u) \\
& =\int_{\mathbb{S}^{d-1}} \int_{[-1,1]} \int_{S_{u, t}} g(t) \sigma_{u, t}(\mathrm{~d} v) \psi(\mathrm{d} t) \sigma(\mathrm{d} u) \\
& =d \kappa_{d} \int_{[-1,1]} g(t) \psi(\mathrm{d} t)
\end{aligned}
$$

since $\sigma_{u, t}\left(S_{u, t}\right)=1$ and $\sigma\left(\mathbb{S}^{d-1}\right)=d \kappa_{d}$. Hence, for $M$ as in the statement of the lemma, the measure $\psi$ is uniquely determined by (12).

Now let $\Theta_{0}$ be a finite, jointly $\mathrm{G}(d)$-invariant, and symmetric measure on $G(d, d-1)^{2}$. A set $B \in \mathcal{B}\left(\left(\mathbb{S}^{d-1}\right)^{2}\right)$ is (for the moment) called small if $(u, v) \in B$ implies $(-u, w) \notin B$ and $(w,-v) \notin B$ for all $w \in \mathbb{S}^{d-1}$. Let $B$ be small. We define

$$
M(B):=\Theta_{0}\left(\left\{\left(u^{\perp}, v^{\perp}\right) \in G(d, d-1)^{2}:(u, v) \in B\right\}\right) .
$$

Clearly, this extends to a finite measure $M$ on $\mathcal{B}\left(\left(\mathbb{S}^{d-1}\right)^{2}\right)$, which is jointly $\mathrm{G}(d)$-invariant and symmetric. From the symmetry, it follows that in (12), we may interchange the first and the second argument of $f$. Therefore, together with (12), the relation

$$
\int_{\left(\mathbb{S}^{d-1}\right)^{2}} f \mathrm{~d} M=\int_{\mathbb{S}^{d-1}} \int_{[-1,1]} \int_{S_{v, t}} f(u, v) \sigma_{v, t}(\mathrm{~d} u) \psi(\mathrm{d} t) \sigma(\mathrm{d} v)
$$

holds for every nonnegative, measurable function $f$ on $\left(\mathbb{S}^{d-1}\right)^{2}$, with the same measure $\psi$. 
Recall that $u(H)$ denotes one of the two unit normal vectors of $H \in G(d, d-$ $1)$. Then for every measurable function $f:\left(\mathbb{S}^{d-1}\right)^{2} \rightarrow[0, \infty)$ which is even in each argument, we have

$$
\int_{G(d, d-1)^{2}} f\left(u\left(H_{1}\right), u\left(H_{2}\right)\right) \Theta_{0}\left(\mathrm{~d}\left(H_{1}, H_{2}\right)\right)=\int_{\left(\mathbb{S}^{d-1}\right)^{2}} f(u, v) M(\mathrm{~d}(u, v)) .
$$

Similarly, let $\Theta_{0}$ be a finite, jointly $\mathrm{G}(d)$-invariant, and symmetric measure on $G(d, 1)^{2}$. For $G \in G(d, 1)$, we denote be $u(G)$ one of the two unit normal vectors parallel to $G$. Clearly, there is a finite, jointly $\mathrm{G}(d)$-invariant and symmetric measure $M$ on $\left(\mathbb{S}^{d-1}\right)^{2}$ such that for every measurable function $f:\left(\mathbb{S}^{d-1}\right)^{2} \rightarrow[0, \infty)$ which is even in each argument, we have

$$
\int_{G(d, 1)^{2}} f\left(u\left(G_{1}\right), u\left(G_{2}\right)\right) \Theta_{0}\left(\mathrm{~d}\left(G_{1}, G_{2}\right)\right)=\int_{\left(\mathbb{S}^{d-1}\right)^{2}} f(u, v) M(\mathrm{~d}(u, v)) .
$$

4. Formulas for general convex bodies. In the following, we assume that $d \geq 3$. The two-dimensional case can be treated with obvious modifications.

We use spherical harmonics, in particular, the Funk-Hecke theorem and the Parseval relation. (For a brief introduction to spherical harmonics, we refer to [8, Appendix], where relevant literature is quoted. A more comprehensive introduction is found in [7, Appendix].) By $\mathcal{H}_{m}^{d}$ we denote the real vector space of spherical harmonics of order $m$ on the unit sphere $\mathbb{S}^{d-1}$. The (finite) dimension of $\mathcal{H}_{m}^{d}$ is denoted by $N(d, m)$. On the space $\mathbf{C}\left(\mathbb{S}^{d-1}\right)$ of continuous real functions on $\mathbb{S}^{d-1}$, we define a scalar product by

$$
(f, g):=\int_{\mathbb{S}^{d-1}} f g \mathrm{~d} \sigma, \quad f, g \in \mathbf{C}\left(\mathbb{S}^{d-1}\right),
$$

where $\sigma$ denotes the spherical Lebesgue measure on $\mathbb{S}^{d-1}$. We write $\sigma\left(\mathbb{S}^{d-1}\right)=$ $\omega_{d}$. Orthogonality on $\mathbf{C}\left(\mathbb{S}^{d-1}\right)$ refers to this scalar product. In each space $\mathcal{H}_{m}^{d}$, we choose an orthonormal basis $\left(Y_{m 1}, \ldots, Y_{m N(d, m)}\right)$. For $f \in \mathbf{C}\left(\mathbb{S}^{d-1}\right)$ and $m \in \mathbb{N}_{0}$, the function

$$
\pi_{m} f:=\sum_{j=1}^{N(d, m)}\left(f, Y_{m j}\right) Y_{m j}
$$

is the image of $f$ under orthogonal projection to the space $\mathcal{H}_{m}^{d}$. The Parseval relation says that

$$
(f, g)=\sum_{m=0}^{\infty} \sum_{j=1}^{N(d, m)}\left(f, Y_{m j}\right)\left(g, Y_{m j}\right)=\sum_{m=0}^{\infty}\left(\pi_{m} f, \pi_{m} g\right) .
$$

Of the Funk-Hecke theorem, we need a consequence, which can be found in Müller [6, Lemma 2, p. 31]. It says that

$$
\int_{S_{v, t}} Y_{m}(u) \sigma_{v, t}(\mathrm{~d} u)=P_{m}(d ; t) Y_{m}(v)
$$


for $m \in \mathbb{N}_{0}, Y_{m} \in \mathcal{H}_{m}^{d}, v \in \mathbb{S}^{d-1}, t \in[-1,1]$, where $P_{m}(d ; \cdot)$ denotes the Legendre polynomial in dimension $d$ of order $m$ (note that the measure $\sigma_{v, t}$ is normalized).

We turn to calculating $I\left(K_{1}, K_{2}, \Theta\right)$ for general convex bodies $K_{1}, K_{2} \in \mathcal{K}^{d}$ and a measure $\Theta \in \mathcal{M}_{d-1}$. From (11), (16), and Lemma 2, we obtain

$$
\begin{aligned}
I\left(K_{1}, K_{2}, \Theta\right) & =\int_{\left(\mathbb{S}^{d-1}\right)^{2}} w_{K_{1}}(u) w_{K_{2}}(v) M(\mathrm{~d}(u, v)) \\
& =\int_{\mathbb{S}^{d-1}} \int_{[-1,1]} \int_{S_{u, t}} w_{K_{1}}(u) w_{K_{2}}(v) \sigma_{u, t}(\mathrm{~d} v) \psi(\mathrm{d} t) \sigma(\mathrm{d} u)
\end{aligned}
$$

with a finite, jointly $\mathrm{G}(d)$-invariant and symmetric measure $M$ on $\left(\mathbb{S}^{d-1}\right)^{2}$ and a finite even measure $\psi$ on $[-1,1]$. Hence, with

$$
g(u):=\int_{[-1,1]} \int_{S_{u, t}} w_{K_{2}}(v) \sigma_{u, t}(\mathrm{~d} v) \psi(\mathrm{d} t),
$$

we get

$$
\begin{aligned}
I\left(K_{1}, K_{2}, \Theta\right) & =\int_{\mathbb{S}^{d-1}} g(u) w_{K_{1}}(u) \sigma(\mathrm{d} u) \\
& =\left(g, w_{K_{1}}\right)=\sum_{m=0}^{\infty}\left(\pi_{m} g, \pi_{m} w_{K_{1}}\right) .
\end{aligned}
$$

With (12), (15), and (18), we obtain

$$
\begin{aligned}
\left(g, Y_{m}\right) & =\int_{\mathbb{S}^{d-1}}\left[\int_{[-1,1]} \int_{S_{u, t}} w_{K_{2}}(v) \sigma_{u, t}(\mathrm{~d} v) \psi(\mathrm{d} t)\right] Y_{m}(u) \sigma(\mathrm{d} u) \\
& =\int_{\left(\mathbb{S}^{d-1}\right)^{2}} Y_{m}(u) w_{K_{2}}(v) M(\mathrm{~d}(u, v)) \\
& =\int_{\mathbb{S}^{d-1}}\left[\int_{[-1,1]} \int_{S_{v, t}} Y_{m}(u) \sigma_{v, t}(\mathrm{~d} u) \psi(\mathrm{d} t)\right] w_{K_{2}}(v) \sigma(\mathrm{d} v) \\
& =\int_{\mathbb{S}^{d-1}}\left[\int_{[-1,1]} P_{m}(d ; t) Y_{m}(v) \psi(\mathrm{d} t)\right] w_{K_{2}}(v) \sigma(\mathrm{d} v) \\
& =\beta_{m}[\Theta]\left(Y_{m}, w_{K_{2}}\right)
\end{aligned}
$$

with

$$
\beta_{m}[\Theta]=\int_{[-1,1]} P_{m}(d ; t) \psi(\mathrm{d} t) .
$$


Therefore,

$$
\pi_{m} g=\sum_{j=1}^{N(d, m)}\left(g, Y_{m j}\right) Y_{m j}=\beta_{m}[\Theta] \sum_{j=1}^{N(d, m)}\left(w_{K_{2}}, Y_{m j}\right) Y_{m j}=\beta_{m}[\Theta] \pi_{m} w_{K_{2}} .
$$

We note that $\beta_{m}[\Theta]=0$ for odd $m$ since $\psi$ is an even measure and the Legendre polynomial $P_{m}(d ; \cdot)$ is an odd function for odd $m$. This finally gives the first part of the following theorem.

Theorem 6. If $K_{1}, K_{2} \in \mathcal{K}^{d}$ and $\Theta \in \mathcal{M}_{d-1}$, then

$$
I\left(K_{1}, K_{2}, \Theta\right)=\sum_{m=0, m \text { even }}^{\infty} \beta_{m}[\Theta]\left(\pi_{m} w_{K_{1}}, \pi_{m} w_{K_{2}}\right),
$$

where $\beta_{m}[\Theta]$ is given by (19).

If $K_{1}, K_{2} \in \mathcal{K}^{d}$ and $\Theta \in \mathcal{M}_{1}$, then

$$
J\left(K_{1}, K_{2}, \Theta\right)=\sum_{m=0, m \text { even }}^{\infty} \beta_{m}[\Theta]\left(\pi_{m} b_{K_{1}}, \pi_{m} b_{K_{2}}\right),
$$

where $b_{K_{i}}$ is the brightness function of $K_{i}$.

To prove the second part of this theorem, we note that a line $G \subset \mathbb{R}^{d}$ parallel to the unit vector $u$ can uniquely be written in the form $G=\operatorname{lin}\{u\}+y$ with $y \in u^{\perp}$. For $G$ represented in this way, we write $u=u(G)$. Let $K_{i} \in \mathcal{K}^{d}$ $(i=1,2)$ and $\Theta \in \mathcal{M}_{1}$. We have

$$
J\left(K_{1}, K_{2}, \Theta\right)=\int_{G(d, 1)^{2}} b_{K_{1}}\left(u\left(G_{1}\right)\right) b_{K_{2}}\left(u\left(G_{2}\right)\right) \Theta_{0}\left(\mathrm{~d}\left(G_{1}, G_{2}\right)\right)
$$

by Lemma 1 , where $\Theta_{0}$ is a finite, jointly $\mathrm{G}(d)$-invariant, and symmetric measure on $G(d, 1)^{2}$. Now the proof of the second part of Theorem 6 can be completed in the same way as that of the first part, just replacing the even function $w_{K_{i}}$ by the even function $b_{K_{i}}$.

Proof of Theorem 2. Assume that $K \in \mathcal{K}^{d}$ is a convex body which satisfies (5) for each $\Theta \in \mathcal{M}_{d-1}$. We only need to consider special measures $\Theta$, of the form

$$
\Theta=\int_{A(d, d-1)^{2}} \mathbb{1}\left\{\left(H_{1}, H_{2}\right) \in \cdot\right\} F\left(\left|\left\langle u\left(H_{1}\right), u\left(H_{2}\right)\right\rangle\right|\right) \mu_{d-1}^{2}\left(\mathrm{~d}\left(H_{1}, H_{2}\right)\right)
$$

with a nonnegative, continuous function $F$. Since both sides of (5) are linear with respect to $\Theta$ (and hence $F$ ), it follows that (5) holds for any continuous function $F$. For such a function $F$, one obtains with the Funk-Hecke formula $([6$, p. 30]) that

$$
\beta_{m}[\Theta]=\omega_{d-1} \int_{-1}^{1} F(|t|) P_{m}(d ; t)\left(1-t^{2}\right)^{\frac{d-3}{2}} \mathrm{~d} t
$$


where $\omega_{d-1}$ is the total spherical Lebesgue measure of the $(d-2)$-dimensional unit sphere.

Now let $k \in \mathbb{N}$ be even, $k \neq 0$. Let $F$ be the restriction of the Legendre polynomial $P_{k}(d ; \cdot)$ to $[-1,1]$. Then $F$ is an even function, and by the orthogonality properties of the Legendre polynomials (see [6, p. 22]), saying that

$$
\int_{-1}^{1} P_{k}(d ; t) P_{m}(d ; t)\left(1-t^{2}\right)^{\frac{d-3}{2}} \mathrm{~d} t \begin{cases}=0 & \text { if } m \neq k, \\ \neq 0 & \text { if } m=k,\end{cases}
$$

we have $\beta_{m}[\Theta]=0$ for $m \neq k$ and $\beta_{k}[\Theta] \neq 0$. Therefore, (4) (where now $\lambda[\Theta]=\beta_{0}[\Theta] \omega_{d}=0$ by $(20)$ ) and (20) give $\pi_{k} w_{K}=0$ for $k \neq 0$ (note that (20) can be applied since $\Theta \in \mathcal{M}_{d-1}$ ). Since $w_{K}$ is an even function, we also have $\pi_{k} w_{K}=0$ for all odd $k$. Now the completeness of the system of spherical harmonics yields that $w_{K}$ is constant. This completes the proof of Theorem 2 .

It is clear that Theorem 4 can be proved similarly.

Proof of Theorem 5. Suppose that $K_{1}$ is of constant width. Then the function $w_{K_{1}}$ is constant and hence $\pi_{m} w_{K_{1}}=0$ for $m \neq 0$ (since constant functions are spherical harmonics of order 0 , and spherical harmonics of different orders are orthogonal). It follows from (20) that

$$
I\left(K_{1}, K_{2}, \Theta\right)=\beta_{0}[\Theta] \omega_{d} W\left(K_{1}\right) W\left(K_{2}\right)=\lambda[\Theta] W\left(K_{1}\right) W\left(K_{2}\right) .
$$

Here we have used that $\pi_{0} f=\omega_{d}^{-1} \int f \mathrm{~d} \sigma$.

Funding Open Access funding enabled and organized by Projekt DEAL.

Open Access. This article is licensed under a Creative Commons Attribution 4.0 International License, which permits use, sharing, adaptation, distribution and reproduction in any medium or format, as long as you give appropriate credit to the original author(s) and the source, provide a link to the Creative Commons licence, and indicate if changes were made. The images or other third party material in this article are included in the article's Creative Commons licence, unless indicated otherwise in a credit line to the material. If material is not included in the article's Creative Commons licence and your intended use is not permitted by statutory regulation or exceeds the permitted use, you will need to obtain permission directly from the copyright holder. To view a copy of this licence, visit http://creativecommons. org/licenses/by/4.0/.

Publisher's Note Springer Nature remains neutral with regard to jurisdictional claims in published maps and institutional affiliations.

\section{References}

[1] Crofton, M.W.: On the theory of local probability, applied to straight lines drawn at random in a plane; the methods used being also extended to the proof of certain new theorems in the integral calculus. Philos. Trans. R. Soc. Lond. 158, 181-199 (1868) 
[2] Cufí, J., Gallego, E., Reventós, A.: Integral geometry about the visual angle of a convex set. arXiv:1906.10374v1

[3] Gardner, R.J.: Geometric Tomography. 2nd edn., Encyclopedia of Mathematics and Its Applications, vol. 58. Cambridge University Press, New York (2006)

[4] Kallenberg, O.: Invariant measures and disintegrations with applications to Palm and related kernels. Probab. Theory Relat. Fields 139, 285-310 (2007). Erratum: Probab. Theory Relat. Fields 139, 311 (2007)

[5] Martini, H., Montejano, L., Oliveros, D.: Bodies of Constant Width. Birkhäuser/Springer, Cham (2019)

[6] Müller, C.: Analysis of Spherical Symmetries in Euclidean Spaces. Springer, New York (1998)

[7] Rubin, B.: Introduction to Radon Transforms. Encyclopedia of Mathematics and Its Applications, vol. 160. Cambridge University Press, New York (2015)

[8] Schneider, R.: Convex Bodies: The Brunn-Minkowski Theory. 2nd edn., Encyclopedia of Mathematics and Its Applications, vol. 151. Cambridge University Press, Cambridge (2014)

[9] Schneider, R., Weil, W.: Stochastic and Integral Geometry. Springer, Berlin (2008)

DANIEl Hug

Department of Mathematics

Karlsruhe Institute of Technology

76128 Karlsruhe

Germany

e-mail: daniel.hug@kit.edu

Rolf SCHNEIDER

Mathematisches Institut

Albert-Ludwigs-Universität

79104 Freiburg i. Br.

Germany

e-mail: rolf.schneider@math.uni-freiburg.de

Received: 26 November 2019 